\title{
Analytical Relationships Between Source-receiver Distances, Redshifts and Luminosity Distances under Pure Modes of Expansion
}

\author{
Denis Michel \\ Université de Rennes1. IRSET. Campus de Villejean. 35000 Rennes cedex, France \\ Email: denis.michel@live.fr
}

\begin{abstract}
The relationships between the redshifts, the luminosity distance and the true distances at the times of light emission and reception, are formally calculated in Euclidean spaces whose expansion is describable using simple functions: proportional to cosmic time, power law or exponential, and for the different assumptions about the origin of the redshift: kinematic, relativistic and wave stretching effects. None of these combinations gives the same results in terms of redshift, of horizon, of distances and of deviation from the Hubble law. This systematic analysis provides the limit cases of expansion scenarios.
\end{abstract}

Keywords: Space expansion, cosmological redshift, cosmological distances, informational horizon.

\section{Introduction}

Different scenarios of expansion have been proposed, based on the predicted physico-chemical maturation stages of the universe, and then tested observationally. But each scenario implies specific approaches to distances. The distance between sources and receivers at the time of light emission or reception are not the distance travelled by light because space expands ahead of and behind the light front. Moreover, these distances cannot be deduced from redshifts without assuming a preconceived distance-redshift diagram under a given mode of expansion. The present paper that is not intended to describe cosmological reality, is aimed at summarizing the dependence of the distances on the mode of expansion, using simple expansion functions. The distance-redshift diagram initiated by Hubble [1] is a central piece of information in cosmology, which can be envisioned as the black box of the universe in which are recorded the past modes of expansion. However, to allow using this diagram for deducing expansion rates, the measurements of the redshifts and distances should not be correlated. Indeed, deducing distances from redshifts would obviously introduce circular distortions in the reasoning. The distance used in the present study is the distance travelled by light, written $D_{\mathrm{L}}$. Among the different measurements of distances in astronomy, summarized in [2], $D_{\mathrm{L}}$ is in theory equivalent to the luminosity distance, based on the decrease of apparent luminosity with distance of objects with known global luminosity. Because of space expansion, $D_{\mathrm{L}}$ is not the distance of the celestial body when the light was emitted since it was shorter, nor the actual distance because the space separating us from the star continued to expand during the travel of light. Hence, mathematical twists are necessary to connect distances and redshifts. These relationships are directly established here for toy universes Euclidean at large scale and steadily expanding according to analytic functions. Let us begin with the basics: the origin of the redshift.

\section{The Main Hypotheses on the Origin of the Redshift}

\subsection{Redshift without Expansion}

Assumptions can be made to explain a generalized redshift without expansion. Relativity would be sufficient to cause distant objects to appear redshifted as a consequence of an apparent slowing of time, but this attractive possibility will not be examined here. Instead, space will be approximated as globally Euclidean, even if it can be locally shaped by gravity. Another hypothesis, called "tired light", assumes that the longer is its flight, the more the light loses its energy by interaction with particles encountered 
along its path (which can originate from the quantum vacuum). As the tired light theory is not based on simple optical rules, it will not be treated here, but this theory curiously uses the same law as the simple exponential expansion [3], which can of course give it a misleading success. Hence, this case will be indirectly treated when studying exponential expansion.

\subsection{The Redshift Caused by Expansion}

The prevailing view is that the cosmological redshift is related to space expansion, but divergences exist about this relation. Two interpretations of the redshift, Doppler effect and wave stretching, have been proposed.

The redshift interpreted as a kinetic Doppler effect. The Hubble Redshift is frequently, perhaps erroneously, interpreted as a Doppler effect conceived conventionally (with a single light emission point but not as the addition of infinitesimal Dopper shifts along the light path postulated in the theory of $[2,4])$. An observer sees a red shift if the light source moved away from him when emitting the observed light. In case of expansion, the distant stars move away from each other, but passively. As mutually receding objects can belong to the same inertial reference frame, the question is whether a passive speed can generate a Doppler effect. With uniform motion, the redshift corresponding to the classical Doppler formula is $z=V_{\text {rec }} / c$ where $V_{\text {rec }}$ is the recession velocity and the relativistic redshift based on the Doppler formula of Einstein [5] is

$$
z_{r}=\sqrt{\frac{c+V_{\text {rec }}}{c-V_{\text {rec }}}}-1
$$

This equation is little used in astrophysics in which recessional velocities can be superluminal $\left(V_{\text {rec }}>c\right)$ [6]. In addition, this formula has been built in special relativity, whereas recession velocities $V_{\text {rec }}$ are generally supposed not uniform.

The redshift interpreted as a stretching of waves during their travel. Before the publication of Hubble [1], Lemaître had shown that wavelengths should follow expansion [7]. For an interval of universe

$$
d s^{2}=d t^{2}-a(t)^{2} d \sigma^{2}
$$

where $d \sigma$ is the element length of a space of radius equal to 1 , the equation of a light beam is

$$
\sigma_{2}-\sigma_{1}=\int_{t_{1}}^{t_{2}} \frac{d t}{a}
$$

where $\sigma_{1}$ and $\sigma_{2}$ are the coordinates of a source and an observer. A beam emitted later at $t_{1}+\delta t_{1}$ and arriving at $t_{2}+\delta t_{2}$ undergoes a shift such that

$$
\frac{\delta t_{2}}{a_{2}}-\frac{\delta t_{1}}{a_{1}}=0
$$

giving

$$
z=\frac{\delta t_{2}}{\delta t_{1}}-1=\frac{a_{2}}{a_{1}}-1
$$

where $\delta t_{1}$ and $\delta t_{2}$ can be considered as the periods at emission and reception respectively [7]. In his article, Lemaitre called this effect a Doppler effect. This term is acceptable if broadly defining the Doppler effect as a wave distortion, but this is not the classical Doppler effect related to the speed of the source.

It will be shown here that these interpretations of the redshift give different results regardless of the type of expansion. In the wave stretching effect of Lemaître, the ratio between the reception and emission wavelengths simply follows the increase of the distance between the source and the receiver which took place during the light flight, between the time point of emission (source and receiver spaced by $D_{\mathrm{E}}$ ) and that of reception (source and receiver spaced by $D_{\mathrm{R}}$ ):

$$
\frac{\lambda^{\mathrm{rec}}}{\lambda}=\frac{D_{\mathrm{R}}}{D_{\mathrm{E}}}
$$


The corresponding redshift $z_{s}$ is the relative distance increase

$$
z_{s}=\frac{D_{\mathrm{R}}-D_{\mathrm{E}}}{D_{\mathrm{E}}} \quad \text { or } \quad D_{\mathrm{R}}=D_{\mathrm{E}}\left(1+z_{s}\right)
$$

\section{Doppler Effects in Uniform Motion}

The simple case of uniform motion is sufficient to perceive the symmetric nature of relative motion and the absence of a static medium. Imagine that a source and a receiver can move relative to one another and in addition, can move relative to an hypothetical static medium supporting light propagation at speed $c$. The source and the receiver recede form each other at speed $v$. At time $t_{\mathrm{E}}$, when spaced from the receiver by $D_{\mathrm{E}}$, the source emits a light beam propagating towards the receiver.

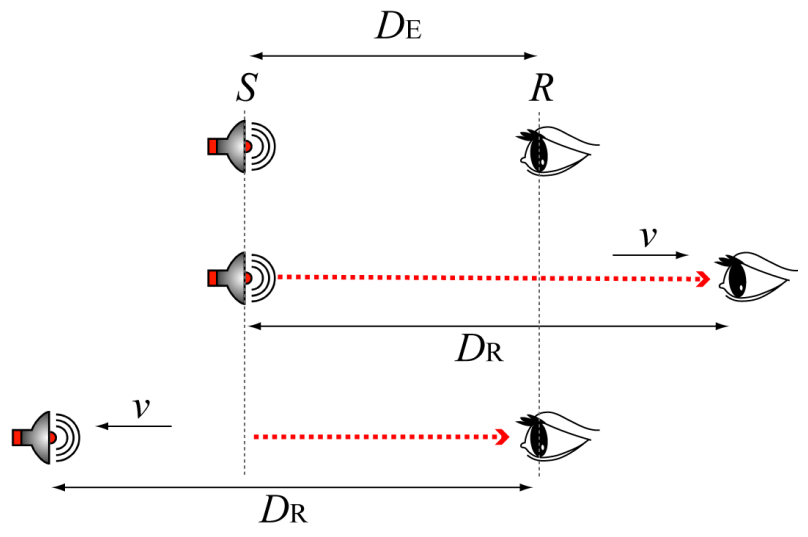

Figure 1. A light pulse is emitted by a source $(S)$ when spaced from a receiver $(R)$ by $D_{\mathrm{E}}$. Just like a ball thrown between two players, light travels through a static medium relatively to which either $S$ (middle line) or $R$ (bottom line), is considered immobile.

\subsection{In an Immobile Medium}

Different results are expected depending on whether this is the source or the receiver which moves relatively to the background medium (Fig.1).

The source is considered immobile relative to the background medium. In this case, light is expected to reach the receiver after crossing a distance $D_{\mathrm{R}}$ (middle line of Fig.1). The duration of the light travel is

$$
t_{\mathrm{R}}-t_{\mathrm{E}}=D_{\mathrm{R}} / c
$$

At $t_{\mathrm{R}}$, the new spacing between the source and the receiver has become

$$
D_{\mathrm{R}}=D_{\mathrm{E}}+v\left(t_{\mathrm{R}}-t_{\mathrm{E}}\right)
$$

Replacing the duration in Eq.(7b) by the value given by Eq.(7a), yields a distance ratio corresponding to a classical Doppler effect

$$
\frac{D_{\mathrm{R}}}{D_{\mathrm{E}}}=\frac{1}{1-\frac{v}{c}}
$$

In case of collinear approach, the same reasoning gives

$$
\frac{D_{\mathrm{R}}}{D_{\mathrm{E}}}=\frac{1}{1+\frac{v}{c}}
$$


The receiver is considered immobile relative to the background medium. Light reaches the receiver at time $t_{\mathrm{R}}$ after crossing a distance $D_{\mathrm{E}}$ (bottom scheme of Fig.1). Hence, the duration of the light travel is

$$
t_{\mathrm{R}}-t_{\mathrm{E}}=D_{\mathrm{E}} / c
$$

Replacing the duration in Eq.(7b) by its value given by Eq.(8a), yields

$$
\frac{D_{\mathrm{R}}}{D_{\mathrm{E}}}=1+\frac{v}{c}
$$

Distance increases in the same ratio as that of the classical Doppler effect. In case of collinear approach, the same reasoning gives

$$
\frac{D_{\mathrm{R}}}{D_{\mathrm{E}}}=1-\frac{v}{c}
$$

These Doppler effects are suitable for the sound that is carried by physical supports, but not for light travelling in vaccuum. Since it is impossible to assign the relative movement to either the source or the observer, it seems natural to take the geometric mean of the two extreme situations of Fig.1 (Eqs (7c)/(8b) and Eqs $(7 d) /(8 c))$. Interestingly, this gives exactly the relativistic Doppler effect. For the recession:

$$
\left\langle\frac{D_{\mathrm{R}}}{D_{\mathrm{E}}}\right\rangle=\sqrt{\frac{c+v}{c-v}}
$$

and for the approach

$$
\left\langle\frac{D_{\mathrm{R}}}{D_{\mathrm{E}}}\right\rangle=\sqrt{\frac{c-v}{c+v}}
$$

\subsection{Without Background Medium}

In the special relativity theory, uniform motion cannot be attributed specifically to one of the relatively moving frames. The total distance crossed by light is not $D_{\mathrm{R}}$ nor $D_{\mathrm{E}}$, but

$$
D_{\mathrm{L}}=c\left(t_{\mathrm{R}}-t_{\mathrm{E}}\right)=\frac{c}{v}\left(D_{\mathrm{R}}-D_{\mathrm{E}}\right)
$$

The precise relationships between $D_{\mathrm{R}}, D_{\mathrm{E}}$ and $D_{\mathrm{L}}$ will be calculated later, but these simple considerations already suggest that a relative speed of light should be defined, not bound to the source or the receiver.

\subsection{Definition of a Relative Speed of Light}

For light following geodesics $(s=0)$, the metric of Friedmann-Robertson is very simple. By rewriting the interval of Eq.(2) in Cartesian coordinates, this metric reads

$$
d s^{2}=(c d t)^{2}-a(t)^{2}\left(d x^{2}+d y^{2}+d z^{2}\right)
$$

where $a(t)$ is the spatial expansion factor depending on time only [8]. For a light beam oriented along the $x$ axis, this line element reduces to

$$
\frac{d x}{d t}=\frac{c}{a(t)}
$$

The ratio would be equivalent to a relative speed if adding a unit of distance to $a(t)$. Accordingly, a time $e^{-1}$ equation is obtained with the Friedmann-Lemaître-Robertson-Walker approach giving $d \chi / d t=c / a(t)$ where $\chi$ is an angle (without dimension) and $a(t)$ is a distance, more appropriately written $R(t)$ in this case. 


\section{Tools and Observables}

\subsection{Nomenclature}

Contrary to usual nomenclature, in cosmology the suffix 0 is not used for the initial condition but for the final condition (present). The following symbols are therefore used to avoid misunderstanding:

$-t_{\mathrm{E}}$ is the date of light emission (initial, generally written $t_{0}$ in other scientific contexts). This date can not be measured directly.

$-t_{\mathrm{R}}$ is the date of reception of the light (final). This is the present age of the universe, written $t_{0}$ by cosmologists. Of course if running time backward, an acceleration becomes a deceleration and vice versa, which may induce some misunderstandings.

- $D_{\mathrm{E}}$ is the initial distance between the star and the telescope (which did not exist yet!) at $t_{\mathrm{E}}$. $D_{\mathrm{E}}$ is not known a priori.

- $D_{\mathrm{R}}$ is the distance between the source and the telescope when light enters it.

- $D_{\mathrm{L}}$ is the distance travelled by light between its emission and its reception. Hence, $D_{\mathrm{L}} / c=t_{\mathrm{R}}-t_{\mathrm{E}}$. $D_{\mathrm{L}}$ is smaller than $D_{\mathrm{R}}$ because the fraction of space already crossed by light continued to stretch until reception. The measurement of $D_{\mathrm{L}}$ is simple, at least in its principle. In a flat 3D space, a source emitted light in all the directions so that the light front progresses as the surface of a sphere $4 \pi D^{2}$ where $D=c t$. As a consequence, the flux of light $I$ detected by an observer at the surface of this sphere corresponds to the $1 / 4 \pi D^{2}$ th of the total light $I_{t o t}$ emitted by the celestial body when it was as it appears in the telescope after redshift correction, giving

$$
D=\sqrt{I_{t o t} / 4 \pi I}
$$

This distance is not based on $z$, even if $z$ is used in practice to assist the measurement, for correcting energy and selecting the range of observable wavelengths to consider. Type Ia supernovae are particularly appropriate for measuring distances by this way given their standard brightness, which greatly helped refining the distance-redshift diagram in the last decades [9].

\subsection{Quantities Measurable in Our Telescopes}

In the theory of Lemaître, the redshift is given by the ratio $D_{\mathrm{R}} / D_{\mathrm{E}}$ (Eq.(6)), but none of these distances is directly measurable, contrary to the distance crossed by light $\left(D_{\mathrm{L}}\right)$. Note that the same light received at $t_{\mathrm{R}}$ is used to measure both the redshift and the distance.

\subsection{Additional Recipes}

$t_{R}$, the present age of the universe, is not known but can be replaced by a constant because it is common to all our measurements. Finally, a very useful tool is the fractional distance $x(t)$ crossed by the front of light between the transmitter and the receiver, defined by the function

$$
x(t)=\int_{\tau=t_{\mathrm{E}}}^{t} \frac{c}{D(\tau)} d \tau
$$

where $D$ is the source-receptor distance. Then, the date of arrival $t_{\mathrm{R}}$ is simply obtained by solving

$$
x\left(t_{\mathrm{R}}\right)=1
$$

\section{Influence of Expansion on Signal Connection}

The relative speeds of light and expansion could limit or even forbid the connection between sources and receivers. Let us examine precisely different cases of expansion. 


\subsection{Uniform Expansion}

Authors suggested a simple mode of universe expansion called $a \propto t$, which follows the cosmic time $[10,11,12]$. When $a(t)=c t, \dot{a}=c$ and $H=1 / t$, expansion follows the age of the universe according to

$$
\frac{D(t)}{D_{\mathrm{E}}}=\frac{t}{t_{\mathrm{E}}}
$$

In fact, with a linear cosmic time, this expansion simply corresponds to the uniform expansion at constant speed $v$. Indeed, Eq.(13) can be re-written

$$
\frac{D(t)}{D_{\mathrm{E}}}=1+\frac{t-t_{\mathrm{E}}}{t_{\mathrm{E}}}
$$

or

$$
D(t)=D_{\mathrm{E}}+\frac{D_{\mathrm{E}}}{t_{\mathrm{E}}}\left(t-t_{\mathrm{E}}\right)
$$

that is

$$
D(t)=D_{\mathrm{E}}+v\left(t-t_{\mathrm{E}}\right)
$$

Uniform expansion means at constant intervals, but not necessarily slow expansion, as the speed can be unlimited. With uniform expansion and given sufficient time, a walker will inevitably cross a road even if stretching at gigantic superluminal speed, whatever his walking speed (eg walk at $c=4$ $\mathrm{km} / \mathrm{h}$ while the road stretches at $v=1,000 \mathrm{~km} / \mathrm{h}$ ). This result is so counterintuitive that it has been popularized as a mathematical game [13]. The relative speed $\left(\right.$ time $\left.^{-1}\right)$ can be written in two manners using the correspondence $D(t)=D_{\mathrm{E}}\left(t / t_{\mathrm{E}}\right)=D_{\mathrm{E}}+v t$,

$$
\frac{d x(t)}{d t}=\frac{c t_{\mathrm{E}}}{D_{\mathrm{E}} t}=\frac{c}{D_{\mathrm{E}}+v t}
$$

whose integration gives a unitless relative position $x$, starting from $x\left(t_{\mathrm{E}}\right)=0$, of

$$
x(t)=\frac{c t_{\mathrm{E}}}{D_{\mathrm{E}}} \ln \frac{t}{t_{\mathrm{E}}}=\frac{c}{v} \ln \left(1+\frac{v t}{D_{\mathrm{E}}}\right)
$$

These functions without ceiling indicate that the walker necessarily reaches $x(t)=1$, even with a very high stretching rate $v$. If the walker is a photon, signals cannot be disconnected in the universe, regardless of the relative speeds of walking and of expansion. The journey can however be very long as its duration follows

$$
\Delta t=\frac{D_{\mathrm{E}}}{v}\left(\mathrm{e}^{v / c}-1\right)
$$

Whatever the relative values of $v$ and $c$ are, there is no disconnection between the different parts of this universe. To try to interrupt this connection, one must change the mode of stretching. Let us examine first a mode of expansion widely used in astrophysics, the geometric progression called "power law".

\subsection{Power law expansion}

This mode of expansion, predicted by calculations based on the theoretical constituents of the universe, satisfies

$$
D(t)=D_{\mathrm{E}}\left(\frac{t}{t_{\mathrm{E}}}\right)^{u}
$$

where $u$ can take different values depending on the maturation stage of the universe described in astrophysics courses: $1 / 2$ for the radiation-dominated era, $2 / 3$ for the matter-dominated era, $1 / 3$ for the stiff fluid, $u=2$ for a minimal condition of inflation satisfying $\ddot{a} / a>0$, etc. The relative position of the walker (photon) is obtained by integration between $t_{\mathrm{E}}$ and $t$ 


$$
\begin{aligned}
x(t) & =\int_{\tau=t_{\mathrm{E}}}^{t} \frac{c}{D_{\mathrm{E}}}\left(\frac{\tau}{t_{\mathrm{E}}}\right)^{-u} d \tau \\
& =\frac{c}{(1-u)} \frac{t_{\mathrm{E}}}{D_{\mathrm{E}}}\left[\left(\frac{t}{t_{\mathrm{E}}}\right)^{1-u}-1\right]
\end{aligned}
$$

Things get interesting:

- (i) For $u<1, x(t)$ increases continuously with $t$ and therefore can exceed 1 without problem. As for the previous modes of expansion, the universe is revealed in its entirety though at different ages.

- (ii) For $u>1$, information cannot cross a virtual limit called horizon

$$
x\left(t_{\infty}\right)=\frac{c t_{\mathrm{E}}}{D_{\mathrm{E}}(u-1)}
$$

The success of the connection depends on the initial conditions of light emission: the time of departure $\left(t_{\mathrm{E}}\right)$ and the distance to be covered as it is at this time $\left(D_{\mathrm{E}}\right)$. Now let us try another accelerated mode of expansion in which time is not raised to a power, but is itself an exponent.

\subsection{Exponential expansion}

With an exponential expansion of rate $H$, the initial distance stretches according to $D(t)=D_{\mathrm{E}} \mathrm{e}^{H \Delta t}$ and the relative speed of light is

$$
\frac{d x(t)}{d t}=\frac{c}{D_{\mathrm{E}}} \mathrm{e}^{-H\left(t-t_{\mathrm{E}}\right)}
$$

whose integration between $t_{\mathrm{E}}$ and $t$, gives his relative position on the source-receiver spacing unit.

$$
x(t)=\frac{c}{H D_{\mathrm{E}}}\left(1-\mathrm{e}^{-H \Delta t}\right)
$$

This position tends asymptotically to a maximum $c / H D_{\mathrm{E}}$, which means that for given values of $H$ and $c$, the success of the connection depends on $D_{\mathrm{E}}$. To reach the target, $c / H D_{\mathrm{E}}$ must exceed 1 , so $D_{\mathrm{E}}$ must be lower than $c / H$. The critical distance $c / H$ is a disconnection point in the expanding universe, that is unreachable because the time of travel

$$
\Delta t=\frac{1}{H} \ln \left(\frac{1}{1-\frac{H D_{\mathrm{E}}}{c}}\right)
$$

would become infinite, and at $t$ infinite, the relative speed $d x(t) / d t$ is zero. This distance is universal in a homogeneously expanding universe and valid from any starting point.

Consequence of the Hubble law $\boldsymbol{V}_{\boldsymbol{r e c}}=\boldsymbol{H} \boldsymbol{D}$. The proportionality between the recession velocity and the distance can be written $d x / d t=H x$, where $H$ is the slope of a straight line. As the slope of a straight line is a constant, the unique solution of the Hubble law is $x(t)=x(0) \mathrm{e}^{H t}$. We see that this expansion is not only a model (of de Sitter), but also just the only possible mathematical solution of the Hubble law as long as $H$ is constant. But it is recommended to write $V_{r e c}=H_{0} D$ to clearly indicate that $H(t)$ may have been different in the past, so the interesting questions are: how much of the universe we can see and how far we have to look for seeing a $H$ different from $H_{0}$. 


\section{Specificities of the exponential mode of expansion.}

- (i) It is an infinite mode of expansion, because $a(t)=a_{0} \mathrm{e}^{H t}$ (Hubble law) has no root, so a universe steadily submitted to exponential expansion has no big bang.

- (ii) This mode of spatial expansion is that of the flat version of the universe of de Sitter [14] (for $k=0$ ), towards which converge at long times the positively curved version $(x(t)=x(0) \cosh H t$ for $k=1)$, and the negatively curved version $(x(t)=x(0) \sinh H t$ for $k=-1)$. After a period of success, the model of exponential expansion of de Sitter was disqualified because it has been shown to not satisfy theoretical predictions based on assumptions of pressure and density of the universe. It is however supposed to be the present mode of expansion driven by vaccum energy since we entered the "dark energy-dominated era" in which $H_{0}$ is a constant $H_{0}=\sqrt{\Lambda / 3}$, where $\Lambda$ is the cosmological constant.

\section{Redshift-Distance Relationships Expected for the Different Modes of Expansion}

Let us examine successively the different modes of expansion and for each one, the two hypothetical causes of redshift : Doppler effect and wave stretching.

\subsection{Power-law Expansion}

\section{Classical Doppler effect.}

$$
D_{\mathrm{E}}=D_{\mathrm{R}}\left(\frac{t_{\mathrm{E}}}{t_{\mathrm{R}}}\right)^{u}
$$

The speed of the source at emission can be deduced from the distance at reception

$$
z_{d}=\frac{1}{c} \frac{d D_{\mathrm{E}}}{d t_{\mathrm{E}}}
$$

that is, taking $D_{\mathrm{R}}$ and $t_{\mathrm{R}}$ as constants

$$
z_{d}=\frac{u D_{\mathrm{R}}}{c} \frac{t_{\mathrm{E}}^{u-1}}{t_{\mathrm{R}}^{u}}=\frac{u D_{\mathrm{E}}}{c t_{\mathrm{E}}}
$$

replacing $t_{\mathrm{E}}$ by $t_{\mathrm{R}}-D_{\mathrm{L}} / c$ and $D_{\mathrm{R}}$ or $D_{\mathrm{E}}$ by their values deduced from $x\left(t_{\mathrm{R}}\right)=1$,

$$
z_{d}=\frac{u}{1-u}\left[\left(1-\frac{D_{\mathrm{L}}}{c t_{\mathrm{R}}}\right)^{u-1}-1\right]
$$

where $t_{\mathrm{R}}$ can be considered as a constant at our time scale.

Wave stretching. The result is straightforward

$$
\frac{D_{\mathrm{R}}}{D_{\mathrm{E}}}=\left(\frac{t_{\mathrm{R}}}{t_{\mathrm{E}}}\right)^{u}=\left(1-\frac{D_{\mathrm{L}}}{c t_{\mathrm{R}}}\right)^{-u}
$$

and

$$
z_{s}=\left(1-\frac{D_{\mathrm{L}}}{c t_{\mathrm{R}}}\right)^{-u}-1
$$

\subsection{The Particular Case $u=1(a \propto t)$}

As mentioned previously, this case merely corresponds to uniform expansion. 


\section{Doppler effect.}

- Classical Doppler effect

The recession velocity for this mode of expansion is simply $D / t$, where $D$ and $t$ vary proportionally,

$$
\frac{D_{\mathrm{E}}}{t_{\mathrm{E}}}=\frac{D_{\mathrm{R}}}{t_{\mathrm{R}}}=v
$$

and

$$
z_{d}=\frac{v}{c}
$$

which supports superluminal velocities.

- Relativistic Doppler effect

Using the special relativistic Doppler equation, the redshift is

$$
z_{r}=\sqrt{\frac{c+v}{c-v}}-1
$$

Wave stretching. By definition for this mode of expansion,

$$
\frac{\lambda^{\mathrm{rec}}}{\lambda}=\frac{D_{\mathrm{R}}}{D_{\mathrm{E}}}=\frac{t_{\mathrm{R}}}{t_{\mathrm{E}}}
$$

and solving $x\left(t_{\mathrm{R}}\right)=1$ yields

$$
\frac{t_{\mathrm{R}}}{t_{\mathrm{E}}}=\mathrm{e}^{D_{\mathrm{R}} / c t_{\mathrm{R}}}=\mathrm{e}^{v / c}
$$

and

$$
z_{s}=\mathrm{e}^{v / c}-1
$$

The two postulated causes of redshift give different results, which are similar only near 0 (for $v<<c$ ). Both predict distance-independent redshifts, which clearly disqualifies this mode of expansion.

\subsection{Exponential Expansion}

\section{Doppler effect.}

- Classical Doppler effect

The speed of the source at the time of light emission is

$$
\frac{d D_{\mathrm{E}}}{d t}=H D_{\mathrm{E}}
$$

whose value is obtained by solving $x\left(t_{\mathrm{R}}\right)=1$,

$$
z_{d}=\frac{H D_{\mathrm{E}}}{c}=1-\mathrm{e}^{-H D_{\mathrm{L}} / c}
$$

As $z_{d}$ is always lower than 1 , for the exponential expansion, the redshift can not exclusively result from a classical Doppler effect.

- Relativistic Doppler effect

$$
z_{r}=\sqrt{\frac{c+H D_{\mathrm{E}}}{c-H D_{\mathrm{E}}}}-1
$$


Wave stretching. The wavelengths are stretched according to

$$
\frac{\lambda^{\mathrm{rec}}}{\lambda}=\frac{D_{\mathrm{R}}}{D_{\mathrm{E}}}=\mathrm{e}^{H \Delta t}=\mathrm{e}^{H D_{\mathrm{L}} / c}
$$

so that

$$
z_{s}=\mathrm{e}^{H D_{\mathrm{L}} / c}-1
$$

whose reciprocal is

$$
D_{\mathrm{L}}=\frac{c}{H} \ln \left(1+z_{s}\right)
$$

\subsection{Mixing Doppler and Stretching Effects for the Exponential Expansion}

The results listed above are derived from formally describable modes of expansion, which are likely to not apply to reality. The expansion mode may have changed and its parameters may have evolved in time. In addition, different modes can be combined in a variety of manners. Let us examine only the case of the exponential expansion. The Doppler and wave stretching redshift functions established above display opposite behaviors near 0, clearly visualized by their series expansion. For the classical Doppler effect,

$$
z_{d} \approx \frac{H_{0} D_{\mathrm{L}}}{c}-\frac{1}{2}\left(\frac{H_{0} D_{\mathrm{L}}}{c}\right)^{2}
$$

and for the wave stretching effect,

$$
z_{s} \approx \frac{H_{0} D_{\mathrm{L}}}{c}+\frac{1}{2}\left(\frac{H_{0} D_{\mathrm{L}}}{c}\right)^{2}
$$

By comparison with the traditional redshift formula $z=H_{0} D / c$, the Doppler effect includes an acceleration parameter, while the wave stretching effect contains a deceleration parameter. For nearby galaxies, the so-called peculiar or ordinary velocities can not be neglected because they can either increase, decrease or cancel the cosmological redshift. Generally, the task of astrophysicists is difficult to disentangle the different interfering sources of wavelength shift, including gravity and peculiar velocities, and to determine the specific contribution of expansion [15]. These technical problems however demonstrate that several causes of redshift can actually interfere. After all, a recession velocity, though passive, could remain a velocity and as such, could contribute as a kinetic Doppler effect to the redshift. Hence, for completeness and by curiosity, let us cumulate the two effects, by taking into account the two hypotheses on the origin of the redshift and two ways to bring them together will be considered.

Combined effects. Assuming, on the one hand, that the dilation of wavelengths caused by kinetic Doppler effect $\left(\lambda^{\text {dopp }}\right)$ is fixed at the emission point and then remains unchanged during the light flight and assuming, on the other hand, that the emission wavelength is expanded during the trip, until received in the form $\lambda^{\text {rec }}$, the cumulative effect would logically read

$$
\frac{\lambda^{\mathrm{rec}}}{\lambda}=\frac{\lambda^{\mathrm{rec}}}{\lambda^{\mathrm{dopp}}} \frac{\lambda^{\mathrm{dopp}}}{\lambda}
$$

using the classical Doppler effect,

$$
z_{d+s}=\left(1+z_{d}\right)\left(1+z_{s}\right)-1=2 z_{s}
$$


Additive effects. Alternatively, as wavelengths are lengths, they should perhaps be handled as such. Adding the relative length increase caused by Doppler effect $z_{d}$ and that caused by stretching $z_{s}$ gives

$$
z_{d+s}=z_{d}+z_{s}
$$

Using the classical Doppler effect,

$$
z_{d+s}=\mathrm{e}^{H D_{\mathrm{L}} / c}-\mathrm{e}^{-H D_{\mathrm{L}} / c}=2 \sinh \frac{H D_{\mathrm{L}}}{c}
$$

The redshift of dual origin of Eq.(40) gives a straighter redshift-distance diagram compared to its individual components (made obvious by the cancellation of the squared terms in Eq.(36)). But if for some reason the Doppler effect predominates for nearby galaxies, a bend would appear in the Hubble diagram for low redshifts, mimicking a recent acceleration for galaxies of redshift below unity. A simple function describing such hypothetic situation is $z=2 \sinh (x) /(1+x)$ with $x=H D_{\mathrm{L}} / c$. It is compared to the observed redshifts of supernovae compiled in [16], represented in Fig.2 in linear coordinates (because a logarithmic scale is poorly appropriate to visualize the straightness of a linear plot). Note that in fact, this bend is currently considered as a recent change of $H$ driven by dark energy [9].

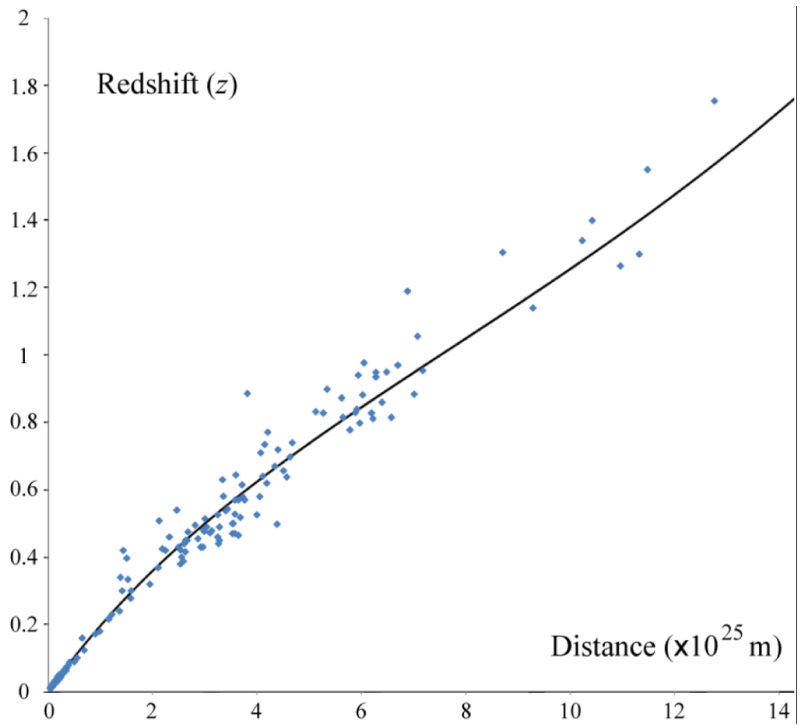

Figure 2. Speculative curve fitting of the distance-redshift supernovae distribution (dots) with an exponential expansion model in which the Doppler effect predominates at short distances while wave stretching predominates at long distances (plain line). The redshift data are from [16] and include 186 supernovae. The fitting parameters are $c=3 \times 10^{8} \mathrm{~m} / \mathrm{s}$ and $H=3.23 \times 10^{-18} / \mathrm{s}$, obtained by conversion of distance modulus $(\mu)$ in a non-logarithmic scale using the relationship $D_{\mathrm{L}}=10^{1+\mu / 5} 10^{-6} \mathrm{Mpc}$.

\section{Distance of a Source Inferred from Its Apparent Distance}

The observed distance of cosmic objects $\left(D_{\mathrm{L}}\right)$ does not correspond to any real distance, but the actual distances can be deduced from $D_{\mathrm{L}}$ provided a mode of expansion is selected.

\section{- For the uniform expansion:}

Since $D_{\mathrm{L}}=c \Delta t$, Eq.(17) gives

$$
D_{\mathrm{E}}=D_{\mathrm{L}} \frac{v / c}{\mathrm{e}^{v / c}-1}
$$


and since $D_{\mathrm{R}}=D_{\mathrm{E}}+v \Delta t$

$$
D_{\mathrm{R}}=D_{\mathrm{L}} \frac{v / c}{1-\mathrm{e}^{-v / c}}
$$

These distances satisfy both the established distances relationship of Eq.(10) and the stretched shift of Eq.(30).

\section{- For the geometric expansion:}

When $x\left(t_{\mathrm{R}}\right)=1$, Eq.(19) gives

$$
D_{\mathrm{E}}=\frac{c t_{\mathrm{R}}-D_{\mathrm{L}}}{1-u}\left[\left(1-\frac{D_{\mathrm{L}}}{c t_{\mathrm{R}}}\right)^{u-1}-1\right]
$$

and

$$
D_{\mathrm{R}}=\frac{c t_{\mathrm{R}}}{1-u}\left[1-\left(1-\frac{D_{\mathrm{L}}}{c t_{\mathrm{R}}}\right)^{1-u}\right]
$$

\section{- For the exponential expansion:}

When $x\left(t_{R}\right)=1$, Eq.(22) gives

$$
H D_{\mathrm{E}}=c\left(1-\mathrm{e}^{-H \Delta t}\right)
$$

with $D_{\mathrm{L}}=c \Delta t$,

$$
D_{\mathrm{E}}=\frac{c}{H}\left(1-\mathrm{e}^{-H D_{\mathrm{L}} / c}\right)=\frac{c z_{d}}{H}
$$

and

$$
D_{\mathrm{R}}=\frac{c}{H}\left(\mathrm{e}^{H D_{\mathrm{L}} / c}-1\right)=\frac{c z_{s}}{H}
$$

\section{Conclusions}

This study expands that of Harrison [15] which rationally derived the relationships between redshift and distance in a purely mathematical manner, under certain types of expansion. A series of simple conclusions can be drawn: For exponential expansion, the traditional redshift formula $z=H_{0} D / c$ is close to $z_{s}=\mathrm{e}^{H_{0} D_{\mathrm{L}} / c}-1$ only in the vicinity of $D=0$ and $z=H_{0} D / c$ is valid only for the invisible actual distance $D_{\mathrm{R}}$ but not for the luminosity distance $D_{\mathrm{L}}$. Whatever the expansion mode, the two postulated origins of the redshift: conventional Doppler and wave stretching effects, always give different results. The mere existence of redshifts higher than 1 is sufficient to rule out the Doppler effect as the sole cause of redshift in the case of the exponential expansion. Finally, the present calculations show that the only two modes of expansion which are capable of generating disconnection barriers, are the exponential expansion (Horizon at $D_{\mathrm{E}}=c / H$ ) and the geometric expansion if $u>1$ (Horizon at $D_{\mathrm{E}}=c t_{\mathrm{E}} /(u-1)$ ). A speculative hypothesis is that as a signal-disconnecting barrier, the horizon could favour the development of life in the universe. In a universe seeded with all the ingredients necessary for the onset of life, the main danger threatening life, that's life itself, with its lack of regard for life forms seen as inferior. Hence, if communication was unrestricted in the universe, a bonus would be given to the rapidly developing forms, but in fact slowly emerging, more complex forms could ultimately be the most effective if sufficient time was given to them. The natural solution to this problem is to partition, at least temporarily, the world into regions disconnected from each other by informational borders. If disconnection limits are conveniently adjusted to the probabilities of life emergence, all forms of life could blossom and grow at their own kinetics, without risk of competition with more advanced forms. The recipe for such a fragmentation is the expansion of the space, provided a mode of extension preventing the signals from reaching other life outbreak sites. As a consequence, if on average, a maximum of one form of life is expected in a connexion sphere, our efforts to not feel alone in the universe might remain in vain. 


\section{References}

1. Hubble, E. 1929. A relation between distance and radial velocity among extra-galactic nebulae. Pnas 15 , 168-173.

2. Hogg, D.W. 2000. Distance measures in cosmology. arxiv.org/abs/astro-ph/9905116

3. Horedt, G. 1973. On the expanding-universe postulate. Astrophys. J. 183, 383-386.

4. Bunn, E.F., Hogg, D.W. 2009. The kinematic origin of the cosmological redshift. Am. J. Phys. 77, 688.

5. Einstein, A. 1905. Zur Elektrodynamik bewegter Körper (On the electrodynamics of moving bodies) Annal. Phys. 17, 891-921.

6. Davis, T.M., Lineweaver, C.H. 2001. Superluminal recession velocities. AIP Conf. Proc. 555, 348.

7. Lemaître G. 1927. Un Univers homog Ấne de masse constante et de rayon croissant rendant compte de la vitesse radiale des nÃlbuleuses extra-galactiques. Annal. Soc. Sci. Bruxelles, A47, 49-59.

8. Einstein, A., de Sitter, W. 1932. On the relation between the expansion and the mean density of the Universe. Proc. Natl. Acad. Sci. U.S.A. 18, 213-214.

9. Perlmutter, S., Schmidt, B.P., Riess, A.G. 2012. Nobel Lecture: Measuring the acceleration of the cosmic expansion using supernovae. Rev. Mod. Phys. 84, 1127-1175.

10. Vigoureux, J.-M., Vigoureux, B. Langlois, M. A New cosmological model. INTECH Open Access Publisher, 2011

11. Cook, R.J., Burns, M.S. 2009. Interpretation of the cosmological metric. Am. J. Phys. 77, 59-66.

12. Melia, F., Shevchuk, A.S.H. 2012. The Rh=ct Universe. Mon. Not. R. Astron. Soc. 419, 2579.

13. Gardner, M. 1988. Time travel and other mathematical bewilderments (New York: W.H. Freeman).

14. de Sitter, W. 1931. The expanding universe. Scientia 49, 1-10.

15. Harrison, E. 1993. The redshift-distance and velocity-distance laws. Astrophys. J. 403, 28-31.

16. Riess, AG, Strolger L.-G., Tonry J., Casertano S., Ferguson H.C., Mobasher B., Challis P., Filippenko A.V., Jha S., Li W., Chornock R., Kirshner R.P., Leibundgut B., Dickinson M., Livio M., Giavalisco M., Steidel C.C., Benitez N., Tsvetanov Z. 2004. Type Ia supernova discoveries at $z>1$ from the Hubble Space Telescope: evidence for past deceleration and constraints on dark energy evolution. Astrophys. J. 607, 665-687. 
Table 1. Summarized consequences of the modes of expansion on the redshift, the horizon and the source-receiver distances. $V_{\text {rec }}$ is the apparent source velocity at emission. $z_{d}$ and $z_{s}$ are the redshifts calculated as Doppler or wave-stretching effects respectively. $D_{\mathrm{E}}$ is the source-receiver distance at emission, $D_{\mathrm{R}}$ is the source-receiver distance at reception and $D_{\mathrm{L}}$ is the apparent distance actually measured. $t_{\mathrm{R}}$ is the present time, approximated as identical for all the measurements.

\begin{tabular}{|c|c|c|c|}
\hline Mode & $a \propto t(u=1)$ & Power law $(u \neq 1)$ & Exponential \\
\hline$D(t)=$ & $D_{\mathrm{E}}\left(\frac{t}{t_{\mathrm{E}}}\right)$ & $D_{\mathrm{E}}\left(\frac{t}{t_{\mathrm{E}}}\right)^{u}$ & $D_{\mathrm{E}} \mathrm{e}^{H\left(t-t_{\mathrm{E}}\right)}$ \\
\hline$\frac{\dot{a}}{a}$ & $\frac{1}{t}$ & $\frac{u}{t}$ & $H$ \\
\hline Horizon & No & $u<1:$ No $\quad u>1:$ for $D_{\mathrm{E}} \geq \frac{c t_{\mathrm{E}}}{u-1}$ & for $D_{\mathrm{E}} \geq \frac{c}{H}$ \\
\hline$V_{\text {rec }}$ & $\frac{D}{t}=v$ & 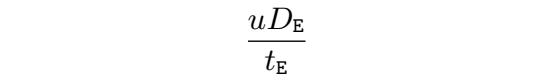 & $H D_{\mathrm{E}}$ \\
\hline$z_{d}$ & $\frac{v}{c}$ & $\frac{u}{1-u}\left[\left(1-\frac{D_{\mathrm{L}}}{c t_{\mathrm{R}}}\right)^{u-1}-1\right]$ & $1-\mathrm{e}^{-H D_{\mathrm{L}} / c}$ \\
\hline$z_{s}$ & $\mathrm{e}^{v / c}-1$ & $\left(1-\frac{D_{\mathrm{L}}}{c t_{\mathrm{R}}}\right)^{-u}-1$ & $\mathrm{e}^{H D_{\mathrm{L}} / c}-1$ \\
\hline$D_{\mathrm{E}}$ & $D_{\mathrm{L}} \frac{v / c}{\mathrm{e}^{v / c}-1}$ & $\frac{c t_{\mathrm{R}}}{1-u}\left(1-\frac{D_{\mathrm{L}}}{c t_{\mathrm{R}}}\right)\left[\left(1-\frac{D_{\mathrm{L}}}{c t_{\mathrm{R}}}\right)^{u-1}-1\right]$ & $\frac{c}{H}\left(1-\mathrm{e}^{-H D_{\mathrm{L}} / c}\right)$ \\
\hline$D_{\mathrm{R}}$ & $D_{\mathrm{L}} \frac{v / c}{1-\mathrm{e}^{-v / c}}$ & $\frac{c t_{\mathrm{R}}}{1-u}\left[1-\left(1-\frac{D_{\mathrm{L}}}{c t_{\mathrm{R}}}\right)^{1-u}\right]$ & $\frac{c}{H}\left(\mathrm{e}^{H D_{\mathrm{L}} / c}-1\right)$ \\
\hline
\end{tabular}

